Tôhoku Math. Journ. 39 (1987), 105-114.

\title{
A FUNDAMENTAL VARIATIONAL LEMMA FOR EXTREMAL QUASICONFORMAL MAPPINGS COMPATIBLE WITH A FUCHSIAN GROUP
}

\author{
KEN-ICHI SAKAN
}

(Received February 18, 1986)

1. Introduction. Let $\Gamma$ be a Fuchsian group acting on the upper half-plane $U$. A holomorphic function $\phi$ on $U$ is called a quadratic differential for $\Gamma$ on $U$ if $\phi(\gamma(z)) \gamma^{\prime}(z)^{2}=\phi(z)$ for every $\gamma \in \Gamma$. Let $\sigma$ be a $\Gamma$-invariant closed subset of the extended real line $\hat{R}=\partial U$, which contains 0,1 and $\infty$ and satisfies $A(\Gamma, \sigma) \neq\{0\}$. Here $A(\Gamma, \sigma)$ denotes the space consisting of all the quadratic differentials $\phi$ for $\Gamma$ on $U$, which are continuously extensible to $\hat{R} \backslash \sigma$ and real on $\hat{R} \backslash \sigma$, and satisfy

$$
\|\phi\|_{U / \Gamma} \equiv \iint_{U / \Gamma}|\phi(z)| d x d y<\infty \text {. }
$$

Let $E$ be a $\Gamma$-invariant measurable, possibly empty, subset of $U$, where $U \backslash E$ has positive area measure and where, if $\operatorname{dim} A(\Gamma, \sigma)=\infty$, then $E / \Gamma$ is assumed to be relatively compact in $\{U \cup(\hat{R} \backslash \sigma)\} / \Gamma$. In what follows, such a configuration $(\Gamma, \sigma, E)$ is said to be admissible.

The "fundamental variational lemma", referred to in the title, is stated as Theorem in Section 4, and is formulated for an arbitrary admissible configuration $\left(\Gamma^{\prime}, \sigma^{\prime}, E^{\prime}\right)$. It is a generalized form of the corresponding one in Reich [7]. Quite recently, in [2], Fehlmann has succeeded in proving it in the case $\Gamma^{\prime}=1$.

In this note, following the method in [2] with some parts modified, we give the proof of our Theorem. In other words, we prove the fundamental variational lemma for an arbitrary admissible configuration $\left(\Gamma^{\prime}, \sigma^{\prime}, E^{\prime}\right)$. In [10], Corollary to our Theorem will be applied to the characterization problem of extremal quasiconformal (qc, for short) mappings compatible with an arbitrary Fuchsian group with an arbitrary dilatation bound. To be more specific, in [10], Corollary to our Theorem will play an important role in characterizing extremal qc mappings within a class $Q \equiv Q(\Gamma, h, \sigma, E, b)$ when $\Gamma^{\prime}, \sigma^{\prime}$ and $E^{\prime}$ are suitably chosen for such a given class $Q$ (see Section 2 for the definitions of $h, b$ and $Q$ ). This is the reason why we formulate our Theorem and Corollary by using notation $\left(\Gamma^{\prime}, \sigma^{\prime}, E^{\prime}\right)$, instead of $(\Gamma, \sigma, E)$, for an admissible configuration. 
The introduction in [2] contains a detailed list of references for the extremal problem for qc mappings mentioned above.

The author would like to express his sincere thanks to Professor Tadashi Kuroda for his helpful advice, and to Professor Richard Fehlmann for valuable information. The author also thanks Professor Masahiko Taniguchi, his colleague Hiromi Ohtake and the referee for helpful comments on the original version of this note.

2. Extremal quasiconformal mappings. In this section, we first collect necessary definitions and notation. For a qc self-mapping $F$ of $U$, we denote its complex dilatation by $\kappa_{F}$, the dilatation of $F$ at the point $z$ by $D_{F}(z) \equiv\left(1+\left|\kappa_{F}(z)\right|\right) /\left(1-\left|\kappa_{F}(z)\right|\right)$ and its maximal dilatation by $K(F)$.

Let $(\Gamma, \sigma, E)$ be an admissible configuration. We denote by $A(\Gamma, \sigma)_{1}$ the set of those $\phi \in A(\Gamma, \sigma)$ with $\|\phi\|_{U / \Gamma}=1$. We note that $A(\Gamma, \sigma) \neq\{0\}$, because $(\Gamma, \sigma, E)$ is admissible. Let $b(z)$ be the so-called dilatation bound function, that is, a non-negative measurable function on $E$, being automorphic for $\Gamma$ and satisfying

$$
\|b\|_{\infty} \equiv \underset{z \in E}{\operatorname{ess} \sup } b(z)<1 .
$$

Furthermore, we are given a quasisymmetric mapping $h: \hat{R} \rightarrow \hat{R}$ induced by a certain qc self-mapping $F_{0}$ of $U$, which is compatible with $\Gamma$ and leaves 0,1 and $\infty$ fixed. We denote by $Q \equiv Q(\Gamma, h, \sigma, E, b)$ the class of all the qc self-mappings $F$ of $U$, which are compatible with $\Gamma$ and satisfy the conditions $\left.F\right|_{\sigma}=\left.h\right|_{\sigma}$ and $\left|\kappa_{F}(z)\right| \leqq b(z)$ a.e. in $E$. In the case $E=\varnothing$, the class is abbreviated to $Q(\Gamma, h, \sigma)$. A mapping $F \in Q$ is said to be extremal if it minimizes the value $\left\|\left.\kappa_{F}\right|_{U \backslash E}\right\|_{\infty}$ within the class $Q$. By Lehto and Virtanen [6, pp. 71-74] and Strebel [11, Satz on p. 469], we see that there exists at least one extremal qc mapping within $Q$, provided that $Q \neq \varnothing$. We put $\sigma^{\prime}=h(\sigma), E_{0}=\{z \in E ; b(z)=0\}$ and, for a fixed $F \in Q$, we introduce

$$
f=F^{-1}, \quad \Gamma^{\prime}=F \Gamma F^{-1}, \quad \kappa=\kappa_{f}, \quad k_{F}=\left\|\left.\kappa_{F}\right|_{U \backslash E}\right\|_{\infty} .
$$

Here we note that $\Gamma^{\prime}$ is determined by $\Gamma$ and $h$ independent of the choice of $F \in Q$. In fact, if $\gamma \in \Gamma$ and $F \in Q$, then $F \circ \gamma \circ F^{-1}$ is identical with the Möbius transformation which sends 0,1 and $\infty$ into $h(\gamma(0)), h(\gamma(1))$ and $h(\gamma(\infty))$, respectively. We denote by $\hat{\kappa}$ the extension of $\left.\kappa\right|_{F\left(\langle U \backslash E) \cup E_{0}\right)}$ to $U$ which satisfies

$$
\hat{\kappa}(z)=k_{F} \kappa(z) / b(f(z)) \quad \text { for } \quad z \in F\left(E \backslash E_{0}\right) .
$$

The following Lemmas 1 and 2 play important roles in the proof of our Theorem. Lemma 1 is due to Gardiner [3]. We can verify Lemma 2 
in the same way as in the proof of [2, Lemma 2.1].

Lemma 1. Suppose that $\operatorname{dim} A(\Gamma, \sigma)<\infty$ and $b(z)=0$ in $E$. Then $F \in Q(\Gamma, h, \sigma, E, b)$ is extremal if and only if

$$
\sup \operatorname{Re} \iint_{U / \Gamma^{\prime}} \hat{\kappa} \phi d x d y=k_{F},
$$

where the supremum is taken over all $\phi \in A\left(\Gamma^{\prime}, \sigma^{\prime}\right)$ with

$$
\|\dot{\phi}\|_{(U \backslash F(E)) / \Gamma^{\prime}} \equiv \iint_{(U \backslash F(E)) / \Gamma^{\prime}}|\phi| d x d y=1 .
$$

REMARK 1. It is known that $\operatorname{dim} A(\Gamma, \sigma)<\infty$ if and only if $\Gamma$ is finitely generated and the set $(\sigma \backslash \Lambda(\Gamma)) / \Gamma$ is finite, where $\Lambda(\Gamma)$ denotes the limit set of $\Gamma$. In particular, $A(\Gamma, \sigma)$ and $A\left(\Gamma^{\prime}, \sigma^{\prime}\right)$ are simultaneously finite dimensional or not.

By Remark 1, we easily have the following Corollary 1 to Lemma 1.

CoROLlaRY 1. Suppose that $\Gamma$ is finitely generated and the set $(\sigma \backslash \Lambda(\Gamma)) / \Gamma$ is finite and that $b(z)=0$ in $E$. In this case, if $F$ is extremal within $Q(\Gamma, h, \sigma, E, b)$, then there exists some $\phi \in A\left(\Gamma^{\prime}, \sigma^{\prime}\right)_{1}$ such that

$$
\begin{aligned}
& \kappa(z)=k_{F} \bar{\phi}(z) /|\phi(z)| \text { a.e. in } U \backslash F(E), \quad \text { and } \\
& \kappa(z)=0 \text { a.e. in } F(E) .
\end{aligned}
$$

LEMmA 2. Let $\Gamma, \sigma, E, h, \Gamma^{\prime}$ and $\sigma^{\prime}$ be as above and let $K \geqq 1$ be a fixed number. Then there exists some $q<1$ satisfying $\|\phi\|_{G_{(E) / \Gamma^{\prime}}} \leqq q$ for every $\phi \in A\left(\Gamma^{\prime}, \sigma^{\prime}\right)_{1}$ and every $G$ in $Q_{K}(\Gamma, h, \sigma)$, where $Q_{K}(\Gamma, h, \sigma)$ means the class consisting of all the $K$-qc self-mappings in $Q(\Gamma, h, \sigma)$.

3. Approximation of $\left(\Gamma^{\prime}, \sigma^{\prime}\right)$. Let $\Gamma^{\prime}$ be a Fuchsian group and let $\sigma^{\prime}$ be a $\Gamma^{\prime}$-invariant closed subset of $\hat{R}$, which contains 0,1 and $\infty$. In this section, we prove some lemmas on an approximation sequence of the configuration $\left(\Gamma^{\prime}, \sigma^{\prime}\right)$ and recall some results.

Let $\left\{\Gamma_{n}^{\prime}\right\}$ be a sequence of finitely generated subgroups of $\Gamma^{\prime}$ and let $\left\{\sigma_{n}^{\prime}\right\}$ be a sequence of closed subsets of $\sigma^{\prime}$. Suppose that the paired sequence $\left\{\left(\Gamma_{n}^{\prime}, \sigma_{n}^{\prime}\right)\right\}$ satisfies the following properties, where $\Lambda_{n}^{\prime}$ denotes the limit set $\Lambda\left(\Gamma_{n}^{\prime}\right)$ of $\Gamma_{n}^{\prime}$ :

(a) $\Gamma_{n}^{\prime} \subseteq \Gamma_{n+1}^{\prime}$ and $\cup \Gamma_{n}^{\prime}=\Gamma^{\prime}$,

(b) $\sigma_{n}^{\prime} \subseteq \sigma_{n+1}^{\prime}$ and the closure of $\cup \sigma_{n}^{\prime}$ is identical with $\sigma^{\prime}$, and

(c) $\sigma_{n}^{\prime}$ is invariant under $\Gamma_{n}^{\prime}, \sigma_{n}^{\prime} \supseteq \Lambda_{n}^{\prime}$ and the set $\left(\sigma_{n}^{\prime} \backslash \Lambda_{n}^{\prime}\right) / \Gamma_{n}^{\prime}$ is finite,

(d) 0,1 and $\infty$ are contained in $\sigma_{1}^{\prime}$.

In this note, we say that such a sequence $\left\{\left(\Gamma_{n}^{\prime}, \sigma_{n}^{\prime}\right)\right\}$ is an approximation 
sequence of the configuration $\left(\Gamma^{\prime}, \sigma^{\prime}\right)$.

As Gardiner [4] states, the following Lemma 3 is not so difficult to show. Here we just sketch the proof.

Lemma 3. For an arbitrarily prescribed configuration $\left(\Gamma^{\prime}, \sigma^{\prime}\right)$, there exists an approximation sequence $\left\{\left(\Gamma_{n}^{\prime}, \sigma_{n}^{\prime}\right)\right\}$ of $\left(\Gamma^{\prime}, \sigma^{\prime}\right)$.

Proof. First we assume that both the group $\Gamma^{\prime}$ and the set $\sigma^{\prime} \backslash \Lambda\left(\Gamma^{\prime}\right)$ are infinite. Let $1, \gamma_{1}, \gamma_{2}, \cdots$ be an enumeration of all the elements of $\Gamma^{\prime}$. We choose a countable dense subset $\left\{a_{1}, a_{2}, \cdots, a_{n}, \cdots\right\}$ of $\sigma^{\prime} \backslash \Lambda\left(\Gamma^{\prime}\right)$. For every $n$, let $\Gamma_{n}^{\prime}$ be the group generated by $\left\{\gamma_{1}, \gamma_{2}, \cdots, \gamma_{n}\right\}$ and let $\sigma_{n}^{\prime}$ be the smallest $\Gamma_{n}^{\prime}$-invariant closed subset of $\hat{R}$, which contains $\left\{a_{1}, a_{2}, \cdots\right.$, $\left.a_{n}, 0,1, \infty\right\}$. As is known, the derived set of $\left\{\gamma(z) ; \gamma \in \Gamma_{n}^{\prime}\right\}$ is identical with $\Lambda\left(\Gamma_{n}^{\prime}\right)$ for every $z \notin \Lambda\left(\Gamma_{n}^{\prime}\right)$ (see Lehner [5, p. 89]). Furthermore, $\Lambda\left(\Gamma^{\prime}\right)$ is identical with the closure of the set consisting of all the fixed points of hyperbolic or parabolic transformations of $\Gamma^{\prime}$ (see [5, p. 104]). In view of these results, we can easily check that $\left\{\left(\Gamma_{n}^{\prime}, \sigma_{n}^{\prime}\right)\right\}$ is an approximation sequence of $\left(\Gamma^{\prime}, \sigma^{\prime}\right)$.

Next we assume that either $\Gamma^{\prime}$ or $\sigma^{\prime} \backslash \Lambda\left(\Gamma^{\prime}\right)$ is finite. In each of these cases, by obvious modification of the above argument, we can obtain such an approximation sequence of $\left(\Gamma^{\prime}, \sigma^{\prime}\right)$.

q.e.d.

LEMMA 4. Let $\left\{\left(\Gamma_{n}^{\prime}, \sigma_{n}^{\prime}\right)\right\}$ be an approximation sequence of $\left(\Gamma^{\prime}, \sigma^{\prime}\right)$. Suppose that $g$ is a qc self-mapping of $U$, which is compatible with $\Gamma^{\prime}$ and where its complex dilatation $\kappa_{g}$ satisfies

$$
\operatorname{Re} \iint_{U / \Gamma^{\prime}} \kappa_{g} \phi d x d y=0 \text { for every } \phi \in A\left(\Gamma^{\prime}, \sigma^{\prime}\right) .
$$

Then we have

$$
\operatorname{Re} \iint_{U / \Gamma_{n}^{\prime}} \kappa_{g} \psi d x d y=0 \text { for every } n \text { and every } \psi \in A\left(\Gamma_{n}^{\prime}, \sigma_{n}^{\prime}\right) \text {. }
$$

Proof. Take an arbitrary element $\psi$ in $A\left(\Gamma_{n}^{\prime}, \sigma_{n}^{\prime}\right)$. Then, by [8, Lemma 8], there exists some $\Phi \in A\left(1, \sigma_{n}^{\prime}\right)$ satisfying $\Theta_{\Gamma_{n}^{\prime}} \Phi=\psi$, where $\Theta_{\Gamma_{n}^{\prime}}$ is the Poincare series operator with respect to $\Gamma_{n}^{\prime}$. Put $\Theta_{\Gamma^{\prime}} \Phi=\phi$. If we note that $A\left(1, \sigma_{n}^{\prime}\right) \subset A\left(1, \sigma^{\prime}\right)$, then, again by [8, Lemma 8], we see that $\phi \in A\left(\Gamma^{\prime}, \sigma^{\prime}\right)$. Since $g$ is compatible with $\Gamma^{\prime}, \kappa_{g}$ satisfies

$$
\kappa_{g}(\gamma(z)) \overline{\gamma^{\prime}}(z) / \gamma^{\prime}(z)=\kappa_{g}(z) \text { for every } \gamma \in \Gamma^{\prime} .
$$

From this, it follows that

$$
\operatorname{Re} \iint_{U / \Gamma_{n}^{\prime}} \kappa_{g} \psi d x d y=\operatorname{Re} \iint_{U} \kappa_{g} \Phi d x d y=\operatorname{Re} \iint_{U / \Gamma^{\prime}} \kappa_{g} \phi d x d y=0 .
$$

We are done, since the choice of $\psi$ is arbitrary.

q.e.d. 
Lemma 5. Let $\left\{\left(\Gamma_{n}^{\prime}, \sigma_{n}^{\prime}\right)\right\}$ be an approximation sequence of $\left(\Gamma^{\prime}, \sigma^{\prime}\right)$ and let $\left\{\phi_{n}\right\}$ be a sequence where $\phi_{n} \in A\left(\Gamma_{n}^{\prime}, \sigma_{n}^{\prime}\right)_{1}$. Then $\left\{\phi_{n}\right\}$ is normal as a family of analytic functions on $U \cup\left(\hat{R} \backslash \sigma^{\prime}\right)$. Moreover, if we take a convergent subsequence, which we call again $\left\{\phi_{n}\right\}$, then $\left\{\phi_{n}\right\}$ converges to some $\phi_{\infty} \in A\left(\Gamma^{\prime}, \sigma^{\prime}\right)$ locally uniformly in $U \cup\left(\widehat{R} \backslash \sigma^{\prime}\right)$ and we have $\left\|\phi_{\infty}\right\|_{U / \Gamma^{\prime}} \leqq 1$.

Proof. Take any $z \in U \cup\left(\hat{R} \backslash \sigma^{\prime}\right)$ and let $N$ be the order of the stabilizer $\Gamma_{z}^{\prime}$ in $\Gamma^{\prime}$ of $z$. Let $V(z)$ be an open neighborhood of $z$ with respect to the topology of $U \cup\left(\hat{R} \backslash \sigma^{\prime}\right)$, such that $\gamma(V(z))=V(z)$ for every $\gamma \in \Gamma_{z}^{\prime}$, and such that $\gamma(V(z)) \cap V(z)$ is empty for every $\gamma \in \Gamma^{\prime} \backslash \Gamma_{z}^{\prime}$. Since $\Gamma_{n}^{\prime} \subset \Gamma^{\prime}$ and $\left\|\phi_{n}\right\|_{U / \Gamma_{n}^{\prime}}=1$, we then have

$$
\iint_{V(z)}\left|\phi_{n}\right| d x d y \leqq N \text { for every } n .
$$

Thus, if we choose an open neighborhood $V_{1}(z)$ of $z$ which is relatively compact in $V(z)$, then, by the mean-value property of analytic functions, we see that $\left\{\phi_{n}\right\}$ is uniformly bounded on $V_{1}(z)$. Hence $\left\{\phi_{n}\right\}$ is a normal family. If a convergent subsequence of $\left\{\phi_{n}\right\}$, which we call again $\left\{\phi_{n}\right\}$, converges to $\phi_{\infty}$, then we easily have

$$
\iint_{\omega^{\prime}}\left|\phi_{\infty}\right| d x d y \leqq \liminf _{n \rightarrow \infty} \iint_{\omega^{\prime}}\left|\phi_{n}\right| d x d y \leqq 1,
$$

where $\omega^{\prime}$ is a fundamental region representing $\left\{U \cup\left(\hat{R} \backslash \sigma^{\prime}\right)\right\} / \Gamma^{\prime}$. Clearly, the limit function $\phi_{\infty}$ is compatible with $\Gamma^{\prime}$ and real on $\hat{R} \backslash \sigma^{\prime}$. Thus we see that $\phi_{\infty} \in A\left(\Gamma^{\prime}, \sigma^{\prime}\right)$.

q.e.d.

Lemmas 6 and 7 below follow from the so-called "main inequality" of Reich and Strebel (see Bers [1] and Strebel [12]).

Lemma 6. Suppose that $f$ and $g$ are two qe self-mappings of $U$, which are compatible with $\Gamma^{\prime}$ and agree on $\sigma^{\prime}$. Then, for every $\phi$ in $A\left(\Gamma^{\prime}, \sigma^{\prime}\right)_{1}$,

$$
1 \leqq \iint_{U / \Gamma^{\prime}}|\phi(z)|\left(\left|1-\kappa_{f}(z) \phi(z) /\right| \phi(z)||^{2} /\left(1-\left|\kappa_{f}(z)\right|^{2}\right)\right) D_{g^{-1}}(f(z)) d x d y .
$$

Lemma 7. Suppose that $f_{n}$ is a Teichmüller mapping with complex dilatation $\left(K_{n}-1\right) \overline{\phi_{n}} /\left(K_{n}+1\right)\left|\phi_{n}\right|$, where $\phi_{n} \in A\left(\Gamma_{n}^{\prime}, \sigma_{n}^{\prime}\right)_{1}$. If $g$ is a qc selfmapping of $U$, which is compatible with $\Gamma_{n}^{\prime}$ and agrees with $f_{n}$ on $\sigma_{n}^{\prime}$, then

$$
K_{n} \leqq \iint_{U / \Gamma_{n}^{\prime}}\left|\phi_{n}(z)\right|\left|1+\kappa_{g}(z) \phi_{n}(z) /\right| \phi_{n}(z)||^{2} /\left(1-\left|\kappa_{g}(z)\right|^{2}\right) d x d y .
$$

4. A fundamental variational lemma. Let $\left(\Gamma^{\prime}, \sigma^{\prime}, E^{\prime}\right)$ be a given admissible configuration. We denote by $N\left(\Gamma^{\prime}, \sigma^{\prime}, E^{\prime}\right)$ the class of all the qc self-mappings $g$ of $U$, which are compatible with $\Gamma^{\prime}$ and where their 
complex dilatations $\kappa_{g}$ satisfy

$$
\begin{gathered}
\kappa_{g}(z)=0 \text { a.e. in } E^{\prime}, \text { and } \\
\operatorname{Re} \iint_{U / \Gamma^{\prime}} \kappa_{g} \phi d x d y=0 \text { for every } \phi \in A\left(\Gamma^{\prime}, \sigma^{\prime}\right) .
\end{gathered}
$$

Now, using lemmas in the previous sections, we prove the following theorem.

THEOREM. Suppose that an admissible configuration $\left(\Gamma^{\prime}, \sigma^{\prime}, E^{\prime}\right)$ is prescribed. Then, for every $g$ in $N\left(\Gamma^{\prime}, \sigma^{\prime}, E^{\prime}\right)$, there exists a qc selfmapping $g^{*}$ of $U$, which is compatible with $\Gamma^{\prime \prime} \equiv g \Gamma^{\prime} g^{-1}$, and satisfies the following properties, where id means the identity automorphism of $U \cup \hat{R}$ :

$$
\begin{aligned}
& g^{* \circ g}=\mathrm{id} \text { on } \sigma^{\prime}, \\
& \kappa_{g^{*}}(z)=0 \text { a.e. in } g\left(E^{\prime}\right), \text { and } \\
& \left\|\kappa_{g^{*}}\right\|_{\infty}=O\left(\left\|\kappa_{g}\right\|_{\infty}^{2}\right) \text { as }\left\|\kappa_{g}\right\|_{\infty} \rightarrow 0 .
\end{aligned}
$$

Proof. Let $g \in N\left(\Gamma^{\prime}, \sigma^{\prime}, E^{\prime}\right)$. Without loss of generality, we may assume that $g$ leaves 0,1 and $\infty$ fixed. We put $\Gamma^{\prime \prime}=g \Gamma^{\prime} g^{-1}, \sigma^{\prime \prime}=g\left(\sigma^{\prime}\right)$, $E^{\prime \prime}=g\left(E^{\prime}\right)$ and consider the class $Q_{0} \equiv Q\left(\Gamma^{\prime \prime},\left.g^{-1}\right|_{\hat{R}}, \sigma^{\prime \prime}, E^{\prime \prime}, 0\right) . Q_{0}$ is not empty, since $Q_{0} \ni g^{-1}$. Thus we can choose an extremal qc mapping $g^{*}$ within the class $Q_{0}$. We show that $g^{*}$ satisfies the required properties. It suffices to prove (4.2). For this purpose, we modify the arguments developed in [2]. By Lemma 3, we have an approximation sequence $\left\{\left(\Gamma_{n}^{\prime}, \sigma_{n}^{\prime}\right)\right\}$ of $\left(\Gamma^{\prime}, \sigma^{\prime}\right)$. Put $\Gamma_{n}^{\prime \prime}=g \Gamma_{n}^{\prime} g^{-1}$ and $\sigma_{n}^{\prime \prime}=g\left(\sigma_{n}^{\prime}\right)$. Choose a fundamental region $\omega^{\prime \prime}$ representing $\left\{U \cup\left(\hat{R} \backslash \sigma^{\prime \prime}\right)\right\} / \Gamma^{\prime \prime}$ and let $S^{\prime \prime}$ be the subset of $\omega^{\prime \prime}$ representing $E^{\prime \prime} / \Gamma^{\prime \prime}$ and put $E_{n}^{\prime \prime}=\cup_{r \in \Gamma_{n}^{\prime \prime}} \gamma\left(S^{\prime \prime}\right)$. We note that $E_{n}^{\prime \prime} \subset E^{\prime \prime}$. Similarly as above, for every $n$, we can choose an extremal qc mapping $G_{n}$ within the class $Q_{n} \equiv Q\left(\Gamma_{n}^{\prime \prime},\left.g^{-1}\right|_{\hat{R}}, \sigma_{n}^{\prime \prime}, E_{n}^{\prime \prime}, 0\right)$. Put $k_{n}=$ $\left\|\left.\kappa_{G_{n}}\right|_{U \backslash E_{n}^{\prime \prime}}\right\|_{\infty}$. Then, by Corollary 1 , there is some $\phi_{n} \in A\left(\Gamma_{n}^{\prime}, \sigma_{n}^{\prime}\right)_{1}$ such that the complex dilatation $\kappa_{n}$ of $g_{n} \equiv G_{n}^{-1}$ satisfies

$$
\begin{aligned}
& \kappa_{n}(z)=k_{n} \overline{\phi_{n}}(z) /\left|\phi_{n}(z)\right| \text { a.e. in } G_{n}\left(U \backslash E_{n}^{\prime \prime}\right), \text { and } \\
& \kappa_{n}(z)=0 \text { a.e. in } G_{n}\left(E_{n}^{\prime \prime}\right) .
\end{aligned}
$$

By definition, we have $Q_{n} \supset Q_{n+1} \supset Q_{0}$ and

$$
\begin{aligned}
& K\left(\left.f\right|_{U \backslash E_{n+1}^{\prime \prime}}\right)=K\left(\left.f\right|_{U \backslash E_{n}^{\prime \prime}}\right) \text { for every } f \in Q_{n+1}, \\
& K\left(\left.f\right|_{U \backslash E_{n}^{\prime \prime}}\right)=K\left(\left.f\right|_{U \backslash E^{\prime \prime}}\right) \text { for every } f \in Q_{0} .
\end{aligned}
$$

Thus we easily see that $k_{n} \leqq\left\|\kappa_{g^{*}}\right\|_{\infty}$ and that $\left\{k_{n}\right\}$ is an increasing sequence. By normality, we may assume that the sequence $\left\{G_{n}\right\}$ converges locally uniformly to a qc self-mapping $G_{\infty}$ of $U$. Then, obviously $G_{\infty}$ is compatible 
with $\Gamma^{\prime \prime}$ and satisfies $\left.G_{\infty}\right|_{\sigma^{\prime \prime}}=\left.g^{-1}\right|_{\sigma^{\prime \prime}}$. By [11, Satz on p. 469], we have

$$
\left|\kappa_{G_{\infty}}(z)\right| \leqq \limsup _{n \rightarrow \infty}\left|\kappa_{G_{n}}(z)\right| \text { a.e. in } U \text {. }
$$

From this, it follows that $\kappa_{G_{\infty}}$ vanishes on $S^{\prime \prime}$. On the other hand, since $G_{\infty}$ is compatible with $\Gamma^{\prime \prime}, \kappa_{G_{\infty}}$ satisfies

$$
\kappa_{G_{\infty}}(\gamma(z)) \overline{\gamma^{\prime}}(z) / \gamma^{\prime}(z)=\kappa_{G_{\infty}}(z) \text { for every } \gamma \in \Gamma^{\prime \prime} .
$$

Since $E^{\prime \prime}=\cup_{r \in \Gamma^{\prime \prime}} \gamma\left(S^{\prime \prime}\right)$, we see that $\kappa_{G_{\infty}}$ vanishes on $E^{\prime \prime}$. Similarly, by (4.4), we have

$$
\left\|\left.\kappa_{G_{\infty}}\right|_{U \backslash E^{\prime}}\right\|_{\infty} \leqq \lim _{n \rightarrow \infty} k_{n} \leqq\left\|\kappa_{g^{*}}\right\|_{\infty} .
$$

Therefore $G_{\infty}$ belongs to $Q_{0}$ and $G_{\infty}$ is extremal within $Q_{0}$. So we have

$$
K\left(G_{\infty}\right)=K\left(g^{*}\right)=\lim _{n \rightarrow \infty} K_{n} \text {, where } K_{n}=\left(1+k_{n}\right) /\left(1-k_{n}\right) .
$$

In order to estimate the numbers $k_{n}$, we let $f_{n}$ to be an extremal qc mapping within the class $Q\left(\Gamma_{n}^{\prime},\left.g\right|_{\hat{k}}, \sigma_{n}^{\prime}\right)$. Its complex dilatation $\tilde{\kappa}_{n}$ is equal to $\widetilde{k}_{n} \widetilde{\phi}_{n} /\left|\tilde{\phi}_{n}\right|$ a.e. in $U$ for some $\tilde{\phi}_{n} \in A\left(\Gamma_{n}^{\prime}, \sigma_{n}^{\prime}\right)_{1}$ and for some constant $\widetilde{k}_{n}, 0 \leqq \widetilde{k}_{n}<1$. Now we use Lemma 6 with $\Gamma^{\prime}$ (resp. $\sigma^{\prime}, \phi, f$ and $g$ ) replaced by $\Gamma_{n}^{\prime}\left(\operatorname{resp} . \sigma_{n}^{\prime}, \phi_{n}^{\prime}, g_{n}\right.$ and $\left.f_{n}\right)$. Then, for $\widetilde{K}_{n} \equiv\left(1+\widetilde{k}_{n}\right) /\left(1-\widetilde{k}_{n}\right)$, we have

$$
1 \leqq \iint_{U / \Gamma_{n}^{\prime}}\left|\phi_{n}(z)\right|\left(\left|1-\kappa_{n}(z) \phi_{n}(z) /\right| \phi_{n}(z)||^{2} /\left(1-\left|\kappa_{n}(z)\right|^{2}\right)\right) \widetilde{K}_{n} d x d y .
$$

Using (4.3), we split the right hand side of (4.5) into an integral over $G_{n}\left(U \backslash E_{n}^{\prime \prime}\right) / \Gamma_{n}^{\prime}$ plus an integral over $G_{n}\left(E_{n}^{\prime \prime}\right) / \Gamma_{n}^{\prime}$. From $K_{n}$ times each of the both sides of the inequality, we subtract $K_{n}\left\|\phi_{n}\right\|_{G_{n}\left(E_{n}^{\prime \prime}\right) / \Gamma_{n}^{\prime}}$. We then have

$$
K_{n}\left(1-\left\|\phi_{n}\right\|_{G_{n}\left(E_{n}^{\prime \prime}\right) / \Gamma_{n}^{\prime}}\right) \leqq \widetilde{K}_{n}\left(1-\left\|\phi_{n}\right\|_{G_{n}\left(E_{n}^{\prime \prime}\right) / \Gamma_{n}^{\prime}}\right)+\left(\widetilde{K}_{n}-1\right) K_{n}\left\|\phi_{n}\right\|_{G_{n}\left(E_{n}^{\prime}\right) / \Gamma_{n}^{\prime}} .
$$

By this inequality, we obtain

$$
K_{n} \leqq \widetilde{K}_{n}+\left(\widetilde{K}_{n}-1\right) K\left(g^{*}\right) /\left(1-\left\|\phi_{n}\right\|_{G_{n}\left(E_{n}^{\prime \prime}\right) / F_{n}^{\prime}}\right),
$$

since $\left\|\phi_{n}\right\|_{G_{n}\left(E_{n}^{\prime \prime}\right) / \Gamma_{n}^{\prime}}<1$ and $K_{n} \leqq K\left(g^{*}\right)$.

Next, in order to estimate $\widetilde{K}_{n}$, we use Lemma 7 with $\phi_{n}$ replaced by $\tilde{\phi}_{n}$ and with $\Gamma_{n}^{\prime}, \sigma_{n}^{\prime}, f_{n}$ and $g$ unchanged. Then we have

$$
\widetilde{K}_{n} \leqq \iint_{U / \Gamma_{n}^{\prime}}\left|\tilde{\phi}_{n}(z)\right|\left|1+\kappa_{g}(z) \tilde{\phi}_{n}(z) /\right| \tilde{\phi}_{n}(z)||^{2} /\left(1-\left|\kappa_{g}(z)\right|^{2}\right) d x d y .
$$

By (4.1) and Lemma 4, we see that the right hand side of (4.7) is not greater than

$$
\left(1+\left\|\kappa_{g}\right\|_{\infty}^{2}\right) /\left(1-\left\|\kappa_{g}\right\|_{\infty}^{2}\right)+2 \operatorname{Re} \iint_{U / \Gamma_{n}^{\prime}}\left(\kappa_{g} \tilde{\phi}_{n} /\left(1-\left|\kappa_{g}\right|^{2}\right)-\kappa_{g} \tilde{\phi}_{n}\right) d x d y .
$$


Since the second term is not greater than $2\left\|\kappa_{g}\right\|_{\infty}^{3} /\left(1-\left\|\kappa_{g}\right\|_{\infty}^{2}\right)$, we easily have

$$
\widetilde{K}_{n} \leqq 1+2\left\|\boldsymbol{\kappa}_{g}\right\|_{\infty}^{2} /\left(1-\left\|\boldsymbol{\kappa}_{g}\right\|_{\infty}\right) .
$$

Thus, by (4.6) and (4.8), we see that

$$
K_{n} \leqq 1+\left(2\left\|\kappa_{g}\right\|_{\infty}^{2} /\left(1-\left\|\kappa_{g}\right\|_{\infty}\right)\right)\left(1+K\left(g^{*}\right) /\left(1-\left\|\phi_{n}\right\|_{G_{n}\left(E_{n}^{\prime \prime}\right) / r_{n}^{\prime}}\right)\right) .
$$

By Lemma 5 , we may assume that $\left\{\phi_{n}\right\}$ converges to $\phi_{\infty}$ locally uniformly in $U \cup\left(\hat{R} \backslash \sigma^{\prime}\right)$, where $\phi_{\infty} \in A\left(\Gamma^{\prime}, \sigma^{\prime}\right)$ and $\left\|\phi_{\infty}\right\|_{U / \Gamma^{\prime}} \leqq 1$. By our construction, we have

$$
\left\|\phi_{n}\right\|_{G_{n}\left(E_{n}^{\prime \prime}\right) / \Gamma_{n}^{\prime}}=\iint_{G_{n}\left(S^{\prime \prime}\right)}\left|\phi_{n}\right| d x d y .
$$

Now we note that $S^{\prime \prime}$ is relatively compact in $U \cup\left(\hat{R} \backslash \sigma^{\prime \prime}\right)$ provided that $\operatorname{dim} A\left(\Gamma^{\prime}, \sigma^{\prime}\right)=\infty$. Thus, as in Fehlmann [2, Appendix], the following equality holds:

$$
\lim _{n \rightarrow \infty} \iint_{G_{n}\left(S^{\prime \prime}\right)}\left|\phi_{n}\right| d x d y=\iint_{G_{\infty}\left(S^{\prime \prime}\right)}\left|\phi_{\infty}\right| d x d y=\left\|\phi_{\infty}\right\|_{G_{\infty}\left(E^{\prime \prime}\right) / \Gamma^{\prime}}
$$

From this equality and (4.9), it follows that

$$
\begin{aligned}
K\left(g^{*}\right) & =\lim _{n \rightarrow \infty} K_{n} \\
& \leqq 1+\left(2\left\|\kappa_{g}\right\|_{\infty}^{2} /\left(1-\left\|\kappa_{g}\right\|_{\infty}\right)\right)\left(1+K\left(g^{*}\right) /\left(1-\left\|\phi_{\infty}\right\|_{\sigma_{\infty}\left(E^{\prime \prime}\right) / \Gamma^{\prime}}\right)\right) .
\end{aligned}
$$

Now, for a fixed number $K>K(g)^{2}$, we use Lemma 2 with $\Gamma$ (resp. $\sigma$, $E, h, \Gamma^{\prime}$ and $\sigma^{\prime}$ ) replaced by $\Gamma^{\prime}$ (resp. $\sigma^{\prime}, E^{\prime}$, id, $\Gamma^{\prime}$ and $\sigma^{\prime}$ ). Since $G_{\infty}\left(E^{\prime \prime}\right)=G_{\infty} \circ g\left(E^{\prime}\right)$ and $G_{\infty} \circ g \in Q_{K}\left(\Gamma^{\prime}\right.$, id, $\left.\sigma^{\prime}\right)$, there exists some $q<1$, which depends on $K$ but not on the choice of $g \in N\left(\Gamma^{\prime}, \sigma^{\prime}, E^{\prime}\right)$ satisfying $K(g)^{2}<K$, such that $\left\|\phi_{\infty}\right\|_{G_{\infty}\left(E^{\prime \prime}\right) / \Gamma^{\prime}} \leqq q$. Hence, by (4.10), we finally have

$$
K\left(g^{*}\right) \leqq 1+(1+K /(1-q)) 2\left\|\kappa_{g}\right\|_{\infty}^{2} /\left(1-\left\|\kappa_{g}\right\|_{\infty}\right) .
$$

This implies (4.2), because we can leave $K$ fixed as $K(g)$ converges to 1 . q.e.d.

Let $L_{\infty}\left(\Gamma^{\prime}\right)$ be the closed linear subspace of $L_{\infty}(U)$ consisting of those $\nu \in L_{\infty}(U)$ which satisfy

$$
\nu(\gamma(z)) \overline{\gamma^{\prime}}(z) / \gamma^{\prime}(z)=\nu(z) \text { for every } \gamma \in \Gamma^{\prime} .
$$

For $\nu \in L_{\infty}\left(\Gamma^{\prime}\right)$ with $\|\nu\|_{\infty}<1$, we denote by $g_{\nu}$ the uniquely determined qc self-mapping of $U$ with complex dilatation $\nu$ and which leaves 0,1 and $\infty$ fixed (see [6, p. 185 and p. 194]). Then we have the following corollary to our Theorem.

CoRollaRY. For a given admissible configuration $\left(\Gamma^{\prime}, \sigma^{\prime}, E^{\prime}\right)$, suppose 
that $\alpha \in L_{\infty}\left(\Gamma^{\prime}\right)$ vanishes on $E^{\prime}$ and that

$$
\operatorname{Re} \iint_{U / \Gamma^{\prime}} \alpha \phi d x d y=0
$$

for every $\phi \in A\left(\Gamma^{\prime}, \sigma^{\prime}\right)$. Then there exists a mapping $\tau$ of an interval $\left(0, t_{0}\right)$ into $L_{\infty}\left(\Gamma^{\prime}\right)$, which satisfies the following properties:

$$
\begin{aligned}
& \tau(t)(z)=0 \text { a.e. in } E^{\prime}, \\
& \|\tau(t)\|_{\infty}<1, \\
& g_{\tau(t)}=\mathrm{id} \text { on } \sigma^{\prime}, \quad \text { and } \\
& \lim _{t \rightarrow 0}\|\tau(t) / t-\alpha\|_{\infty}=0 .
\end{aligned}
$$

Proof. If $\alpha=0$, then we have only to choose $\tau(t) \equiv 0$. So we may assume that $\|\alpha\|_{\infty}>0$. From our hypotheses, it follows that $g_{t \alpha} \in$ $N\left(\Gamma^{\prime}, \sigma^{\prime}, E^{\prime}\right)$ for $t<1 /\|\alpha\|_{\infty}$. Thus we can apply our Theorem to $g_{t \alpha}$ for $t<1 /\|\alpha\|_{\infty}$. Then there exists some qc self-mapping $g_{t}^{*}$ of $U$, which is compatible with $g_{t \alpha} \Gamma^{\prime} g_{t \alpha}^{-1}$ and satisfies

$$
\begin{aligned}
& g_{t}^{*} \circ g_{t \alpha}=\mathrm{id} \text { on } \sigma^{\prime}, \\
& \kappa_{g_{t}^{*}}(z)=0 \text { a.e. in } g_{t \alpha}\left(E^{\prime}\right), \text { and } \\
& \left\|\kappa_{g_{t}^{*}}\right\|_{\infty}=O\left(\|t \alpha\|_{\infty}^{2}\right)=O\left(t^{2}\right) \text { as } t \rightarrow 0 .
\end{aligned}
$$

If we put $\tau(t)=\kappa_{g_{t}^{*} \circ g_{t \alpha}}$, then we can easily check that $\tau(t)$ satisfies the required properties.

q.e.d.

ADDENDUM. The above corollary is a restatement of Proposition in [9] which was used in the proof of Theorem 2 in [9]. The proof of the proposition was incomplete. The gap in the argument in [9] was on p. 89, lines 8-9, where $\left\{\lambda \in L_{\infty}(V, G) ;\|\lambda\|_{\infty}=\left\|T_{2}(\lambda)\right\|\right\}$ is not a linear space in general. By the above corollary, however, Theorem 2 in [9] is nonetheless valid.

\section{REFERENCES}

[1] L. BERs, A new proof of a fundamental inequality for quasiconformal mappings, J. Analyse Math. 36 (1979), 15-30.

[2] R. Fehlmann, On a fundamental variational lemma for extremal quasiconformal mappings, Comment. Math. Helv. 61 (1986), 565-580.

[3] F.P. Gardiner, On partially Teichmüller Beltrami differentials, Michigan Math. J. 29 (1982), 237-242.

[4] F.P. Gardiner, Approximation of infinite dimensional Teichmüller spaces, Trans. Amer. Math. Soc. 282 (1984), 367-383.

[5] J. LeHNER, Discontinuous groups and automorphic functions, Mathematical Surveys 18, Amer. Math. Soc., Providence, Rhode Island, 1964. 
[6] O. Lehto and K. I. Virtanen, Quasiconformal mappings in the plane, Springer-Verlag, Berlin-Heidelberg-New York, 1973.

[7] E. REICH, Quasiconformal mappings with prescribed boundary values and a dilatation bound, Arch. Rational Mech. Anal. 68 (1978), 99-112.

[8] K. SaKan, On quasiconformal mappings compatible with a Fuchsian group, Osaka J. Math. 19 (1982), 159-170.

[9] K. SAKAN, On extremal quasiconformal mappings compatible with a Fuchsian group with a dilatation bound, Tôhoku Math. J. 37. (1985), 79-93.

[10] K. SAKAN, Necessary and sufficient conditions for extremality in certain classes of quasiconformal mappings, J. Math. Kyoto Univ. 26 (1986), 31-37.

[11] K. StREBel, Ein Konvergenzsatz für Folgen quasikonformer Abbildungen, Comment. Math. Helv. 44 (1969), 469-475.

[12] K. StReBeL, On quasiconformal mappings of open Riemann surfaces, Comment. Math. Helv. 53 (1978), 301-321.

DePaRTMENT OF MATHEMATICS

OSAKA City UNIVERSITY

SUGIMOTO, SUMIYOSHI-KU

OSAKA 558

JAPAN 